\title{
Time de acesso vascular e terapia infusional: satisfação da equipe de saúde em hospital pediátrico
}

RESUMO | Objetivos: Conhecer a satisfação e qualidade do serviço sobre time de acesso vascular e terapia infusional (TAVTI) e identificar a importância do TAVTI para a equipe de saúde. Metodo: Trata-se de uma pesquisa de campo exploratória, descritiva, com abordagem quantitativa e qualitativa. Aplicamos formulários para 149 profissionais. Resultados: Dos participantes, 46,97\%(n:70) refere total satisfação frente ao TAVTI; a minoria 0,67\%(n:1) demonstra insatisfação total; a maioria considera o serviço muito importante $71,14 \%$ (n:106) e excelente atuação para 44,96\%(n:67). Apenas 1,34\%(2) dos profissionais considerou a qualidade do trabalho regular. Dos participantes, 126 declararam: serviço humanizado; seguro para o paciente; necessita de capacitação e treinamento; material seguro; período integral. Conclusão: A atuação do TAVTI demonstra ser efetiva. Constatouse a satisfação da equipe de saúde e a importância na pediatria devido à preservação do acesso venoso e o desejo dos profissionais em manter o TAVTI período integral. Frente ao percentual de qualidade considerado, excelente e ótimo.

Palavras-chaves: Dispositivos de Acesso Vascular; Pessoal de Saúde; Satisfação do Paciente; Pediatria; Gestão da Qualidade.

\begin{abstract}
Objectives: To know the satisfaction and quality of service on the vascular access and infusional therapy team (TAVTI) and to identify the importance of TAVTI for the health team. Methodo: This is an exploratory, descriptive field research, with a quantitative and qualitative approach. We apply forms to 149 professionals. Results: Of the participants, 46.97\% (n: 70) reported total satisfaction with TAVTI; the minority $0.67 \%$ ( $n: 1)$ shows total dissatisfaction; the majority considered the service very important $71.14 \%$ ( $n$ : 106) and excellent performance for $44.96 \%$ ( $n$ : 67). Only $1.34 \%$ (2) of the professionals considered the quality of work to be regular. Of the participants, 126 declared: humanized service; safe for the patient; needs qualification and training; safe material; Full time. Conclusion: The performance of TAVTI proves to be effective. The satisfaction of the health team and the importance in pediatrics were found due to the preservation of venous access and the desire of professionals to maintain TAVTI full time. In view of the percentage of quality considered, excellent and great.
\end{abstract}

Keywords: Vascular Access Devices; Health Personnel; Patient Satisfaction; Pediatrics; Quality Management.

RESUMEN | Objetivos: Conocer la satisfacción y calidad del servicio del equipo de acceso vascular y terapia infusional (TAVTI) e identificar la importancia de TAVTI para el equipo de salud. Metodo: Se trata de una investigación de campo exploratoria, descriptiva, con enfoque cuantitativo y cualitativo. Aplicamos formularios a 149 profesionales. Resultados: De los participantes, el 46,97\% (n: 70) reportaron satisfacción total con TAVTI; la minoría 0,67\% (n: 1) muestra total insatisfacción; la mayoría consideró el servicio muy importante $71,14 \%$ ( $n$ : 106) y excelente desempeño para el $44,96 \%$ ( $n$ : 67 ). Solo el $1,34 \%$ (2) de los profesionales consideró regular la calidad del trabajo. De los participantes, 126 declararon: servicio humanizado; seguro para el paciente; necesita cualificación y formación; material seguro; período integral. Conclusión: La actuación de TAVTI demuestra ser eficaz. La satisfacción del equipo de salud y la importancia en pediatría se encontraron debido a la preservación del acceso venoso y el deseo de los profesionales de mantener TAVTI a tiempo completo. En vista del porcentaje de calidad considerado, excelente y óptimo.

Palabras claves: Dispositivos de Acceso Vascular; Personal de Salud; Satisfacción del Paciente; Pediatría; Gestión de la Calidad.

\section{Wilza Cabral Rodrigues da Silva}

Enfermeira do Time de acesso vascular e Terapia Infusional do Hospital Infantil Cândido Fontoura. Mestre em Ciências da Saúde pelo Programa de Pós-Graduação em Ciências da Saúde. Especialista em Emergência e UTI Saúde da Criança e Adolescente pelo Instituto da criança ICR-USP. São Paulo (SP). Brasil. ORCID: 0000-0003-2353-8332

\section{Sandra Alves Neves Araújo}

Enfermeira do Time de acesso vascular e Terapia Infusional do Hospital Infantil Cândido Fontoura. Mestre em Ciências da Saúde pela Universidade Guarulhos. Especialista em Segurança do Paciente pela Fundação Oswaldo Cruz.

ORCID: 0000-0002-7341-0911

\section{Maria do Carmo da Costa}

Enfermeira do Time de acesso vascular e Terapia Infusional do Hospital Infantil Cândido Fontoura.Enfermeira do Hospital Infantil Sabará. Especialista Neonotalogia pela Universidade Bandeirante. São Paulo (SP). Brasil. ORCID : 0000-0003-4187-4321

\section{Ana Maria Abrunhosa Vasconcelos}

Enfermeira. Especialista em Segurança do Paciente pela Fundação Oswaldo Cruz. Diretoria Técnica de Saúde do Hospital Infantil Cândido Fontoura. Preceptor de estágio da Universidade Cruzeiro do Sul. ORCID: 0000-0003-4786-4298

Recebido em: 04/11/2020

Aprovado em: 10/12/2020

\section{Andreia Lopes Siqueira}

Técnica de enfermagem do Time de acesso vascular e Terapia Infusional do Hospital Infantil Cândido Fontoura. Graduação em enfermagem pela Universidade Nove de Julho. . São Paulo (SP). Brasil.

ORCID: 0000-0002-5302-8299

\section{Michele de Souza Bizari}

Auxiliar de enfermagem do Time de acesso vascular e Terapia Infusional do Hospital Infantil Cândido Fontoura. Graduação em enfermagem pelaUniversidade Camilo Castelo Branco. Especialização em pediatria e neonatologia pela AVM Faculdades Integradas. . São Paulo (SP). Brasil. ORCID: 0000-0002-1228-5213 
INTRODUÇÃO

A punção venosa é caracterizada pela colocação de um dispositivo no interior do vaso venoso, podendo ou não ser fixado à pele. Esse procedimento requer cuidados e controle periódico durante a hospitalização. ${ }^{(1)}$ A observação da punção é extremamente importante, pois se o local da inserção mostrar-se com sinais e sintomas de complicações, a terapia deve ser suspensa, mesmo que o cateter esteja instalado por um período inferior a $24 \mathrm{hs}$.

As veias periféricas tornam-se gradualmente danificadas por infusões de antibióticos, com alta osmolaridade, nutrições parenterais e agentes quimioterápicos. Além disso, pacientes pediátricos frequentemente referem que as punções venosas e o uso dos cateteres são alguns dos fatos mais traumáticos de sua hospitalização. Uma criança com difícil acesso venoso pode ser submetida de 12 a 20 tentativas de inserção do cateter em 4 semanas de internação. ${ }^{(2)} \mathrm{O}$ limite máximo recomendado é de até três tentativas de punção. ${ }^{(2)}$

Concomitante ao avanço tecnológico da terapia intravenosa, o enfermeiro deve mostrar sua competência técnica e científica para o desenvolvimento da prática da punção de veias periféricas e administração de medicamentos, considerando que é responsabilidade da enfermagem a decisão sobre a escolha de locais, tipos de dispositivos, calibres, documentação de instalação, manutenção de curativos e prevenção de complicações. ${ }^{(3)}$

A terapia intravenosa é um dos procedimentos que requer inovações na prática do enfermeiro, respaldada em análise científica que contribui para melhorar os resultados assistenciais. Além disso, é de responsabilidade do enfermeiro estabelecer o acesso venoso periférico, incluindo o cateter central de inserção periférica (PICC), conforme o Parecer COREN-SP CAT n. ${ }^{\circ}$ 020/2010(4) e, respaldo em evidências cientificas para garantir a segurança do paciente e dos profissionais e ser realizada mediante a elaboração efetiva da Siste- matização da Assistência de Enfermagem, prevista na Resolução COFEN n. ${ }^{0358 / 09 . ~(5) ~}$

O TAVTI foi constituído a partir das Diretrizes Práticas para Terapia Intravenosa da Infusion Nurses Society ${ }^{(6)}$, proposto à Direção de Enfermagem da instituição estudada, na qual o TAVTI é constituído por enfermeiros, médicos, farmacêuticos, técnicos e auxiliares de enfermagem. Sua formação com profissionais especializados tem sido uma estratégia recomendada para prestar cuidados à criança que necessita de um acesso vascular. Desempenha sua atividade exclusivamente para inserção, manutenção, retirada do Peripherally Inserted Central Catheter (PICC), acesso venoso periférico difícil, soluciona e discute as complicações relacionadas à terapia infusional, além de realizar consultoria e educação permanente. ${ }^{(7,8)}$

Em resumo, o TAVTI trabalha em conjunto para rever, analisar, implementar, educar e sustentar as melhores práticas, enfatizando a segurança do paciente além de controlar custos através da redução do desperdício. ${ }^{(8)}$

O TAVTI é estruturado para atender as necessidades dos pacientes com eficácia e alta qualidade. A terapia de infusão segura prevê resultados positivos para o paciente, incluindo prevenção de complicações e satisfação do paciente e do profissional de saúde, quando é dada atenção a quatro áreas principais: seleção apropriada do paciente, eficaz educação do paciente, atendimento meticuloso ao paciente e avaliação e monitoramento abrangentes e comunicação e colaboração interprofissional. (9)

Ademais, destacamos a importância da existência de protocolo institucional relacionado ao Time de Acesso Vascular e Terapia Infusional (TAVTI), reforçando os cuidados a serem prestado a fim de garantir a assistência de enfermagem segura, sem risco ou danos ao paciente pediátrico causados por negligência, imperícia ou imprudência, conforme Art. 12 do Código de Ética dos Profissionais de Enfermagem. ${ }^{(10)}$

Sendo assim, o enfermeiro do TAVTI é referência para avaliar os dispositivos intravasculares regularmente, inclusive a necessidade de instalação e permanência do dispositivo mais adequado para a terapia infusional conforme as necessidades da criança, garantindo uma internação segura e com qualidade assistencial. ${ }^{(6,11)}$

Ao falar de qualidade, é comum fazermos relação à sociedade com nossa expectativa individual, que também é influenciada pela expectativa de qualidade da sociedade como um todo; então, ao falar de qualidade, diz-se de um produto ou serviço e se este atendeu ou não às nossas expectativas ou mesmo se as superou. ${ }^{(12)}$

A qualidade em saúde é uma responsabilidade dos profissionais e uma expectativa dos pacientes. ${ }^{(13)}$ Em relação à satisfação, pode possibilitar ao enfermeiro implementar mudanças na prática e contribuir à gestão com propostas de melhorias na qualidade da assistência em saúde. ${ }^{(14)}$ Ou seja, sua satisfação através da qualidade do serviço que lhes é prestado, de uma equipe multidisciplinar com condições tecnológicas para garantir uma boa assistência e um administrador que satisfaça as suas necessidades de maneira segura e lucrativa. ${ }^{(15)}$

É imperioso o reconhecer a terapia intravenosa (TIV) como processo amplo e complexo no trabalho multidisciplinar com visão e suporte institucional e no estabelecimento dos pontos críticos deste processo. Contudo, passíveis de monitoramento e alinhados às novas tendências de boas práticas na terapia intravenosa, com foco na qualidade da assistência de enfermagem.

Após três meses de implantação, propomos conhecer a satisfação dos técnicos, auxiliares de enfermagem, enfermeiros e médicos sobre o TAVTI, bem como identificar a importância desse serviço para o cuidado de enfermagem, uma vez que as inovações fazem parte do cuidado diário das crianças.

Diante do exposto, o presente trabaIho tem a finalidade de permitir traçar medidas que busquem aprimorar a assistência de enfermagem visando verificar se há necessidade de adaptações ou alterações neste serviço. Buscou-se responder ao seguinte questionamento: Qual a 
satisfação dos técnicos, auxiliares de enfermagem, enfermeiros e médicos sobre o TAVTI e, a qualidade e importância deste serviço no hospital infantil? Tem-se como objetivos conhecer a satisfação e a qualidade do serviço sobre o Time de Acesso Vascular e Terapia Ifusional e identificar a sua importância para a equipe de saúde.

\section{MÉTODO}

Trata-se de uma pesquisa de campo exploratória, descritiva, com abordagem quantitativa, qualitativa e transversal realizada em um hospital infantil localizado na cidade de São Paulo. Fizeram parte da pesquisa 149 profissionais que prestam assistência direta aos pacientes. Utilizamos como critério de elegibilidade, amostra não probabilística intencional composta pelos indivíduos que aceitaram participar livremente do estudo.

Aplicamos o formulário caracterizando os participantes e, através da escala de Likert(16), para identificar o grau de satisfação, qualidade do serviço e categorizando o conteúdo atraves da analise de Bardin (17), juntamente à assinatura do TCLE, entre junho e julho de 2020, em respeito aos aspectos éticos das pesquisas com seres humanos ${ }^{(18,19)}$, após parecer favorável da plataforma Brasil, sob no 4.098.120 e autorização da Diretoria de Enfermagem do hospital pediátrico. Quanto aos critérios de inclusão, aceitar participar da pesquisa, atuar na prestação de cuidados diretos ao paciente pediátrico e; critérios de exclusão: recusar-se a participar da pesquisa; não prestar cuidados diretos ao paciente; não assinar o TCLE. Foram excluídos os profissionais de saúde que prestavam cuidados indiretos à criança.

Frente ao atual cenário que vivenciamos nos serviços de saúde com a pandemia, houve redução no quadro dos profissionais no período da coleta de dados, fato no qual consiste na limitação do estudo.

\section{RESULTADOS}

Passamos a apresentar os resultados quantitativos das variáveis de interesse, o que possibilita a caracterização da amostra. Aceitaram participar 149 profissionais da área da saúde. Desses, 87,91\%(n:131) do gênero feminino e, $11,40 \%(n: 17)$ masculino. Apenas 0,67\% (n:1) não informou.

Quanto à equipe da saúde, 20,80\%(n:31) da categoria médica, $34,22 \%(n: 51)$ auxiliares de enfermagem, 20,13\%(n:30) técnicos de enfermagem e, 24,83\%(n:37) enfermeiros. Em relação à faixa etária, 6,04\%(n:9) encontrava-se entre 20 e 30 anos e 38,92\%(n:58) segue entre 51 e 60 anos.
São formados há 10 anos, 12,75\%(n:19) profissionais e, 75,16\%(n:112) está entre 21 e 30 anos de formação. Apenas 8,05\%(n:12) atua há mais de 40 anos na pediatria, tratando-se de uma equipe, em sua maioria, experiente. Não informaram 2,68\%(n:04) o tempo de atuação na área.

Nesta etapa apresentamos resultados de estatística descritiva das variáveis de interesse, o que possibilita conhecer a satisfação, a importância do TAVTI para a equipe de saúde e a qualidade do serviço.

Verificamos na tabela acima que 46,97\%(n:70) da equipe de saúde refere
Tabela 1. Distribuição segundo satisfação, importância, qualidade do TAVTI junto a equipe de saúde. São Paulo, SP, Brasil, 2020

\section{Satisfação do TAVTI}

Totalmente satisfeito

n $\%$

Satisfeito

Parcialmente satisfeito

70

Nem satisfeito e nem insatisfeito

Parcialmente insatisfeito

Insatisfeito

Totalmente insatisfeito

Total

\begin{tabular}{lcc}
\hline Importância do TAVTI & $\mathbf{n}$ & $\%$ \\
\hline Muito importante & 106 & 71,14 \\
\hline Importante & 40 & 26,84 \\
\hline Bom & 3 & 2,01 \\
\hline Razoavelmente importante & 0 & 0 \\
\hline Moderadamente importante & 0 & 0 \\
\hline É pouco importante & 0 & 0 \\
\hline Não é importante & 0 & 0 \\
\hline Total & 149 & 100 \\
\hline Qualidade do TAVTI & $\mathbf{n}$ & $\%$ \\
\hline Excelente & 67 & 44,96 \\
\hline Ótimo & 52 & 34,89 \\
\hline Bom & 28 & 18,79 \\
\hline Indiferente & 0 & 0 \\
\hline Regular & 2 & 1,34 \\
\hline Ruim & 0 & 0 \\
\hline Muito ruim & 0 & 0 \\
\hline Total & 149 & 100 \\
\hline
\end{tabular}


total satisfação frente ao TAVTI. Sentem-se satisfeitos 39,59\%(n:59) e, 10,73\%(n:16) revelaram parcialmente satisfeitos. Considera-se nem satisfeitos e nem insatisfeitos, apenas 2,68\%(n:4) profissionais. A minoria demonstra insatisfação parcial e total $0,67 \%(n: 1)$, respectivamente.

A grande maioria dos integrantes da equipe de saúde refere o TAVTI muito importante 71,14\%(n:106) e importante $26,84 \%(n: 40)$ para o serviço. Apenas 2,10\%(n:3) considera o trabalho bom.

O serviço do TAVTI destaca-se pela excelência para 44,96\%(n:67) dos profissionais, ótimo 34,89\%(n:52) e bom 18,79\%(n:28). Desenvolve trabalho regular para 1,34\% (n:02).

A categorização qualitativa expressa a opinião dos participantes referente ao desempenho do TAVTI. Exemplificamos abaixo as cinco áreas temáticas identificadas a partir do fragmento discursivo:

Exemplificamos abaixo as cinco áreas temáticas identificadas a partir do fragmento discursivo:

A equipe de saúde expressa a importância da atuação do TAVTI em Período integral, 69,04\% (n:87):

"[...] período integral junto com a enfermagem."

"Manter plantão noturno de $12 \mathrm{~h}$, porque às vezes temos acesso difícil á noite, ou seja, na madrugada [...]"
"O time precisa funcionar aos finais de semana e feriado."

"Permanência deste time, longo prazo."

"[...] gostaria que funcionasse $24 \mathrm{~h} . "$ "[...] aumentar o grupo para não sobrecarregar. Acompanhamento integral no período noturno."

Os participantes apontaram se tratar de um Serviço humanizado, 26,19\% (n:33):

"[...] continuar com todo carinho que vocês prezam ao atender o paciente."

"OTAVTI gera menos sofrimento [...]" "[...] time auxilia cada caso, tomando a melhor conduta, gerando menos sofrimento para o tratamento." "O time é um sucesso e trabalham com amor."

"[...] essencial para a criança e de grande ajuda ao serviço médico."

No que concerne à Segurança do paciente, $24,60 \%(n: 31)$ :

"[...] identificação de possíveis eventos adversos ocasionadas no processo de infusão medicamentosa."

"[...] capacitação de toda equipe para um olhar clinico e eficiente na prevenção de eventos adversos."

Figura 1. Distribuição segundo a caracterização do desempenho do TAVTI junto à equipe de saúde. São Paulo, SP, Brasil, 2020
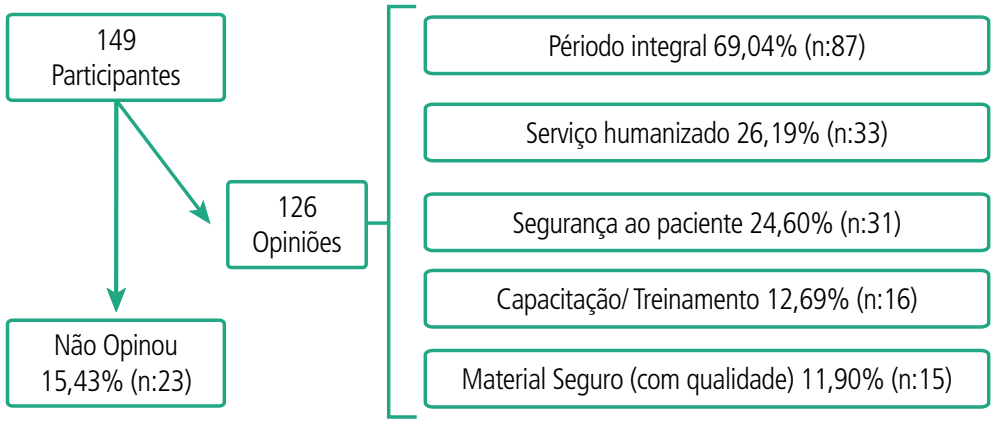

"[...] contribui muito para melhoria da nossa assistência o time apresenta uma resposta rápida quando acionada o que melhora a rapidez e eficácia do atendimento."

"[...] excelente trabalho que ajuda muito no tratamento e melhora dos clientes."

"Qualidade"

"[...] reduziu muito os casos de flebite." "A maior e melhor iniciativa em prol do cuidado a criança."

"[...] o TAVTI visa a qualidade e a segurança do paciente.

Os participantes do estudo referiram-se ainda à Capacitação/Treinamento, $12,69 \%$ (n:16):

"[...] treinamento periódico para manutenção do acesso venoso."

"[...] gostaria que o time realizasse treinamento para equipe de enfermagem no período noturno [...]"

"Aprimoramento e acompanhamento da equipe in loco [...]"

"[...] treinamento para implementação do cuidado."

"[...] treinamento em relação a manutenção e fixação do acesso."

Por último, a necessidade do uso de Material seguro, 11,90\% (n:15):

"[...] melhorar a qualidade dos materiais de punção [...]"

"[...] aquisição de matérias mais seguro, de melhor qualidade."

"O material esta de péssima qualidade."

"As coberturas não colam."

"[...] precisamos cobrir com Micropore [...]"

"Que o material de fixação seja de melhor qualidade."

"[...] a necessidade de trocar o material."

"[...] evitar desperdício de material." "Realmente são nota 10, apesar de se virarem sem equipamento de ponta $[\ldots]^{\prime \prime}$ 


\section{DISCUSSÃO}

A implantação do TAVIT contribui para a qualidade da assistência através da implementação das melhores práticas com o estabelecimento de protocolos, monitorizando indicadores estratégicos e atuando com outras equipes para otimizar processos e reduzir complicações. Assim, os protocolos, guindelines e pacotes de cuidados (bundles) são estratégias para promover a qualidade e a segurança do paciente. ${ }^{(7)}$

Neste cenário, a segurança remete para a qualidade e requer competência profissional para que seja capaz de impactar sobre a qualidade dos cuidados e permitir que o profissional passe a dar um significado amplo ao cuidado, na perspetiva da compaixão, da dignidade e do respeito para com o doente, aspectos esses que podem ser melhorados pela análise das experiências e pelo entendimento da satisfação da crianças e familiares. ${ }^{(20)}$

Os serviços de saúde que trabalham com TAVIT apresenta resultados positivos nos indicadores de qualidade da prestação de cuidados de enfermagem, com redução nas taxas de flebite e de infecção relacionada a cateter, bem como no número de punções venosas, conduzindo à inserção mais precoce do PICC e à maior satisfação do doente e da equipe assistencial. ${ }^{(21,22,23)}$ Mullan 2001, considera o cuidado com qualidade é um cuidado genuíno, com amor envolvendo preocupação com as necessidades do paciente ${ }^{(24)}$

Diante desse contexto, é importante afirmar que a qualidade da assistência e a segurança do paciente caminham juntas. Assim, de acordo com Institute of Medicine (IOM), a segurança do paciente é um dos domínios para a qualidade da assistência, ou seja, garantir a segurança do paciente é uma das maneiras de assegurar a qualidade da assistência. ${ }^{(25)} \mathrm{A}$ segurança do paciente culmina na preocupação a respeito da qualidade prestada no cuidado ao paciente. $\mathrm{O}$ cuidado não pode ser considerado de alta qualidade a menos que seja seguro ${ }^{(26)}$.

A qualidade na assistência e a segu- rança do paciente estão relacionadas, assim, para a Organização Pan-Americana de Saúde (OPAS), os princípios relacionados com a qualidade na assistência de enfermagem são o ponto de partida para a introdução de programas de melhoria no cuidado ao paciente, assim como, contribuem com a diminuição de risco durante o cuidado ${ }^{(25)}$.

O Ministério da Saúde considera que

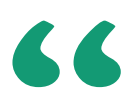

\section{Em relação à qualidade dos materiais} disponíveis para os cuidados, é essencial a seleção de uma película estéril e transparente, pois esse permite a frequente visualização do local de inserção do Cateter Venoso Periferico (CVP), para a identificação precoce de manifestações clínicas de uma possível complicação.

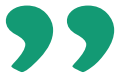

as várias formas de construção de conhecimentos, habilidades e práticas, as ações educativas em si, devem ser compreendidas para além do sentido clássico da aquisição de conhecimentos especifico e profissional, com a finalidade de modificar a realidade, construr e criar novas formas de gestão dos processos de trabalho. ${ }^{(27)}$

Diante dos resultados adquiridos, a educação continuada na enfermagem é imprecindível, pois é indispensável a performance de uma equipe capacitada e com conhecimento para realizar suas atribuições. Para tanto, faz se necessário preparar um plano de capacitação e, por conseguinte, a ampliação da competência individual e profissional dos envolvidos. ${ }^{(28)}$

Em relação à qualidade dos materiais disponíveis para os cuidados, é essencial a seleção de uma película estéril e transparente, pois esse permite a frequente visualização do local de inserção do Cateter Venoso Periferico (CVP), para a identificação precoce de manifestações clínicas de uma possível complicação. ${ }^{(29)}$ Em síntese, um cuidado de qualidade deve estar alicerçado em evidências científicas, alinhado com a segurança na utilização de materiais apropriados, além da tecnologia disponível, como o ecógrafo, enquanto recurso tecnológico de imagem, indicado para avaliar o diâmetro da veia antes da inserção do PICC, contribuindo para reduzir o risco de trombose venosa profunda pela seleção adequada do calibre do cateter compatível com a veia ${ }^{(30)}$.

A ocorrência de eventos adversos e de queixas técnicas relacionadas a produtos para a saúde sugere a existência de riscos que podem representar ameaças à saúde da criança, além de comprometer a execusão do procedimento pela equipe de saúde. Situações que podem estar associadaa à baixa qualidade do produto; ao seu uso de forma inadequada (erros de procedimento); a fatores inerentes à pessoa (no caso de evento adverso); bem como a fatores do próprio produto, muitas vezes já indicados no processo de registro na Agência Nacional de Vigilância Sanitária (ANVISA). ${ }^{31,32)}$ 


\section{CONCLUSÃO}

A presença do TAVTI demonstra ser efetiva em crianças com punção venosa difícil. Constatou-se a satisfação da equipe de saúde e a importância para a pediatria de- vido à preservação do acesso venoso. A capacitação e participação dos profissionais são as maneiras mais adequadas para se alcançar a segurança do paciente, pois as mudanças transformam o ser humano, seja na área pessoal, profissional ou institucional. Vale ressaltar o desejo da maioria dos profissionais em manter o TAVTI em período integral. Frente ao percentual de qualidade considerado, excelente e ótimo.

\section{Referências}

1. Torres MM, Andrade DD, Santos CB. Punção venosa periférica: avaliação de desempenho dos profissionais de enfermagem. Revista Latino - Am Enfermagem [Internet]. 2005 mai-jun [acesso em 12 mar 2019]; 13(3):299-304. Disponível em: $<$ www.scielo.br/pdf/rlae/v13n3a03.pdf>

2. Vendramin P. Cateter central de inserção periférica (CCIP) em crianças. Centro de estudos e pesquisas, Boletim Científico [Internet]. 2007 mar [acesso em 12 mar 2019]; 7. Disponível em: <http://portal.samaritano.com.br/pt/interna.asp?page $=1$ \&idpagina $=294>$

3. Pereira RCC, Zanetti ML, Ribeiro KP. Tempo de permanência do dispositivo periférico in situ, relacionado ao cuidado de enfermagem em pacientes hospitalizados. Medicina Ribeirão Preto [Internet]. 2011 jan/mar [acesso em 12 mar 2019]; 34(1):79-84. Disponível em: <http://www.revistas.usp.br/rmrp/article/view/1195>. 4. Brasil. Conselho Regional de Enfermagem de São Paulo. Parecer COREN-SP CAT n. ${ }^{\circ}$ 020/2010. Dispõe sobre Terapia intravenosa [Internet]. São Paulo (SP): COREN; 2010 [acesso em 19 mar 2019]. Disponivel em: <https://portal.coren.sp.gov.br/site/ default/file/parecer_coren_sp_2010_20.pdf>.

5. Brasil. Conselho Federal de Enfermagem. Resolução n. ${ }^{\circ}$ 358, de 15 de outubro de 2009. Dispõe sobre sistematização da assistência de enfermagem e implementação do processo de enfermagem em ambientes públicos e privados, em que ocorre o cuidado profissional de enfermagem, e da outras providencias. Diario Oficial da união, 23 out, 2009.

6. Infusion Nurses Society - INS Brasil. Diretrizes práticas para terapia infusional. $127 p, 2018$.

7. Miller-Hoover S. Pediatric Central Line - Bundle Implementation and Outcomes. Journal of Infusion Nursing [Internet]. 2011 [acesso em 19 mar 2019];34(1):36-48 Disponivel em: <https://pubmed.ncbi.nlm.nih.gov/21239950/>.

8. Silva AA, Feijó RDF, Lessa SS. Infecção da corente sanguínea associada ao uso de cateteres vasculares. $4^{a}$ ed.rev.e ampl.. São Paulo: APECIH; 2016. 232p.

9. Caguiaoa J, et al. Hands: standardised intravascular practice based on evidence. British Journal of Nursing [Internet]. 2012 [acesso em 19 mar 2019]; 21(14-IV suplem.):S4-S11. Disponível em: <https://pubmed.ncbi.nlm.nih.gov/23252175/>. 10. Brasil. Conselho Federal de Enfermagem. Resolução n. ${ }^{\circ} 311$, de 08 de fevereiro de 2007. Aprova a reformulação do Código de Ética dos Profissionais de Enfermagem. Diário oficial da União 13 de fev de 2007.

11. Couto AS, Matos C, Stangler Ml. Importância do time de acesso vascular no cenário pediátrico hospitalar. Lume UFRGS [Internet]. 2018 [acesso em $19 \mathrm{mar}$ 2019]. Disponível em: <https://lume.ufrgs.br/bitstream/handle/10183/182373/001074390.pdf?sequence=1 \&isAllowed=y $>$.

12. Silva L. FN, da Silva MA. Satisfação do Paciente como estratégia de marketing hospitalar para conquistar clientes. Estudos [Internet]. 2014 [acesso em 25 mar 2019]; 41:87-100. Disponível em: <http://seer.pucgoias.edu.br/index.php/estudos/ article/view/3810>.

13. Albuquerque CIRA, Madeira GS, Capanema IVL, Ferreira JS. Qualidade em saúde: evolução e desafios no contexto brasileiro. Qualidade em Saúde. Universidade de Ciencias Empresariales y Sociales Facultad de Ciencias de la Sáude Asignatura: Gerenciación Estratégica y Marketing en Salud. Buenos Aires 2017.

14. Dorigan G, Guirardello EB. Satisfação do paciente em uma unidade de gastroenterologia. Acta Paul Enferm [Internet]. 2010 [acesso em 25 mar 2019] 23(4):500-505. Disponível em: <https://www.scielo.br/scielo.php?script=sci_abstract\&pid=S0103-21002010000400009\&lng=pt\&nrm=iso>.

15. Pereira GS, Pereira SS. A importância da qualidade do serviço na gestão hospitalar. Rev. Eletrôn. Atualiza Saúde [Internet]. 2015 jan/jun [acesso em 25 mar 2019];1(1). Disponivel em: <http://atualizarevista.com.br/wp-content/uploads/2015/01/pdf>.

16. Dalmoro M, Vieira KM. Dilemas na construção de escalas tipo likert: o número de itens e a disposição influenciam nos resultados? Revista Gestão Organizacional [Internet]. 2013 [acesso em 20 mar 2019]; 6(esp). Disponivel em: <http://www. anpad.org.br/admin/pdf/EPQ-A1615.pdf>.

17. Bardin L. Análise de conteúdo. São Paulo: Edições 70; 2010.

18. Brasil. Ministério da Saúde. Conselho Nacional de Saúde. Resolução n. ${ }^{\circ} 466$, de 12 de dezembro de 2012. Trata de pesquisas em seres humanos e atualiza a Resolução n. ${ }^{\circ}$ 196/1996. Brasília (DF): MS/CNS; 2012.

19. Brasil. Ministério da Saúde. Conselho Nacional de Saúde. Resolução n. ${ }^{\circ}$ 510/2016/CNS/MS. Brasília. DOU nº 98, sec1. 44-46, 2016.

20. Gorski LA. The Infusion Therapy Standards of Practice. Home Healthcare Now [Internet]. 2017 jan [acesso em 23 set 2020]; 35(1):10-18. Disponível em: <https://portaldeboaspraticas.iff.fiocruz.br/biblioteca/the-2016-infusion-therapy-standards-of-practice/>.

21. Alonso E. Impact of the creation of a short-term central venous catheter (CVC) follow up and improvement multidisciplinary team. 2016. IN: 4 th World Congress on Vascular Access WoCoVA 2016. June 22-24, 2016, Lisbon, Pt. The Journal of Vascular Access, 17(4), e 28. doi: 10.5301/jva.5000587

22. Cechinel RB, Zimerman RA, Nunes DS, Veçossi DSG, Mesquita K, Galo L, Sukiennik TCT. The effect of implementation of an adult's hospitalwide vascular access team on central line-associated bloodstream infections. 2016. IN: 4 th World Congress on Vascular Access WoCoVA 2016. June 22-24, 2016, Lisbon, Pt. The Journal of Vascular Access, 17(4), e4. doi: 10.5301/jva.5000587

23. Espírito-Santo FRF, Schneidwind KPDR, Vega GCMD, Passalacqua AL, Oliveira CAS, Cruz AP. Implementation of vascular access medical team as a way to reach best assistance in a general hospital. 2016. São Paulo, Brazil. IN: 4 th World Congress on Vascular Access WoCoVA 2016. June 22-24, 2016, Lisbon, PT. The Journal of Vascular Access, 17(4), e15. doi: 10.5301/jva.5000587

24. Mullan FA. Founder of quality assessment encounters a troubled system firsthand. Interview. Health Affairs. 2001;20(1):137-141. doi: 10.1377/hlthaff.20.1.137 25. Grajales RAZ, et al. La calidad y seguridad del paciente: elementos conceptuales. IN: Cometto MC. et al. Enfermería y seguridade de los pacientes. Organización Panamericana de la Salud, 2011. p. 139-163

26. Moss F, Barach P. Quality and safety in health care: a time of transition: safe health care. Quality Heath Care [Internet]. 2002 [acesso em 08 out 2020]. Disponível em: $<$ www.qualityhealthcare.com $>$.

27. Brasil. Ministério da Saúde. Secretaria-Executiva (Org.). Educação Permanente em Saúde: um movimento instituinte de novas práticas no Ministério da Saúde, 2014.

28. Carvalho JD. A importancia da educação continuada em enfermagem. Internet 2020. Rev Saberes [Internet]. 2020 jun [acesso em 26 nov 2020]; 12(1). Disponivel em: <https://facsaopaulo.edu.br/wp-content/uploads/sites/16/2020/06/A-IMPORTANCIA-DA-EDUCA\%C3\%87\%C3\%830-CONTINUADA-NA-ENFERMAGEM. pdf>.

29. Oliveira ASS. Intervenção nas práticas aos enfermeiros na prevenção aeflebites em pessoas portaaoras ae cateteres venosos periféricos: Um estudo de investigação-ação. IN: 2014. (Tese de doutoramento).

30. Chopra V, Anand S, Hickner A, Buist M, Rogers MA, Saint S, Flanders SA. Risk of venous thromboembolism associated with peripherally inserted central catheters: A systematic review and meta-analysis. Lancet. 2013; 382(9889):311-325. doi: 10.1016/S0140-6736(13)60592-9

31. Brasil. Ministério da Saúde. Agência Nacional de Vigilância Sanitária. Gestão da tecnologia biomédica. Cap. 3, 2011.

32. Hofmeister MGS, Vicente MG, Fornazier CRO et al. Manual de Tecnovigilância: abordagens para a vigilância sanitária de produtos para saúde comercializados no Brasil. ANVISA/MS. Brasilia, 2008. 